\title{
A $1 \mu \mathrm{W}$ radiation-hard front-end in a $0.18 \mu \mathrm{m}$ CMOS process for the MALTA2 monolithic sensor
}

\author{
F. Piro, Student Member, IEEE, P. Allport, I. Asensi, I. Berdalovic, D. Bortoletto, C. Buttar, R. Cardella, E. \\ Charbon, Fellow, IEEE, F. Dachs, V. Dao, D. Dobrijevic, M. Dyndal, L. Flores, P. Freeman, A. Gabrielli, L. \\ Gonella, T. Kugathasan, M. LeBlanc, K. Oyulmaz, H. Pernegger, P. Riedler, M. van Rijnbach, H. Sandaker, A. \\ Sharma, C. Solans, W. Snoeys, Senior Member, IEEE, T. Suligoj, J. Torres, and S. Worm
}

\begin{abstract}
Monolithic pixel sensors integrate the sensor matrix and readout in the same silicon die, and therefore present several advantages over the more largely used hybrid detectors in highenergy physics. In this paper, a low-power, radiation-hard frontend circuit for monolithic pixel sensors, designed to meet the requirements of low noise and low pixel-to-pixel variability, the key features to achieve high detection efficiencies, is presented. The sensor features a small collection electrode to achieve a small capacitance $(<5 \mathrm{fF})$ and allows full CMOS in-pixel circuitry. The circuit is implemented in the $180 \mathrm{~nm}$ CMOS imaging technology from the TowerJazz foundry and integrated in the
\end{abstract}

Manuscript received January 25, 2022; revised xxxxxxxx xx, xxxx; accepted $\operatorname{xxxxxxxx} x x, \operatorname{xxxx}$. Date of publication $\operatorname{xxxxxxxx} \mathrm{xx}, \mathrm{xxxx}$; date of current version $\mathrm{xxxxxxxx} \mathrm{xx}, \mathrm{xxxx}$.

F. Piro is with the CERN Experimental Physics Department, Geneva, Switzerland and with the Advanced Quantum Architecture Laboratory (AQUA), École Polytechnique Fédérale de Lausanne, Switzerland (e-mail: francesco.piro@cern.ch).

P. Allport, P. Freeman and L. Gonella are with the Department of Physics and Astronomy, University of Birmingham, United Kingdom (e-mail: allport@cern.ch; patrick.freeman@cern.ch; laura.gonella@cern.ch).

I. Asensi, F. Dachs, V. Dao, D. Dobrijevic, M. Dyndal, L. Flores, A. Gabrielli, T. Kugathasan, M. LeBlanc, H. Pernegger, P. Riedler, M. van Rijnbach, A. Sharma, C. Solans and W. Snoeys are with the CERN Experimental Physics Department, Geneva, Switzerland (e-mail: ignacio.asensi@cern.ch; florian.dachs@cern.ch; valerio.dao@cern.ch; dominik.dobrijevic@cern.ch; mateusz.dyndal@cern.ch; leyre.flores@cern.ch; andrea.gabrielli@cern.ch; thanushan.kugathasan@cern.ch; matt.leblanc@cern.ch; heinz.pernegger@cern.ch; petra.riedler@cern.ch; milou.van.rijnbach@cern.ch; abhishek.sharma@cern.ch; los.solans@cern.ch; walter.snoeys@cern.ch).

I. Berdalovic was with the CERN Experimental Physics Department, Geneva, Switzerland. He is now with the Faculty of Electrical Engineering and Computing, University of Zagreb, Croatia (e-mail: ivan.berdalovic@ fer.hr).

D. Bortoletto is with the Department of Particle Physics, University of Oxford, United Kingdom (e-mail: daniela.bortoletto@physics.ox.ac.uk).

C. Buttar is with the Faculty of Physics and Astronomy, University of Glasgow, United Kingdom (e-mail: craig.buttar@glasgow.ac.uk).

R. Cardella was with the CERN Experimental Physics Department. He is now with the Department of Nuclear and Particle Physics, University of Geneva, Switzerland (e-mail: roberto.cardella@unige.ch).

E. Charbon is with the Advanced Quantum Architecture Laboratory (AQUA), École Polytechnique Fédérale de Lausanne, Switzerland (e-mail: edoardo.charbon@epfl.ch).

K. Oyulmaz is with the Department of Physics, Bolu Abant Izzet Baysal University, Turkey (e-mail: kaan.yuksel.oyulmaz@cern.ch).

H. Sandaker is with the Department of Physics, University of Oslo, Norway (e-mail: heidi.sandaker@fys.uio.no).

T. Suligoj is with the Faculty of Electrical Engineering and Computing, University of Zagreb, Croatia (e-mail: tomislav.suligoj@fer.hr).

J. Torres is with the Department of Electronics Engineering, University of Valencia, Spain (e-mail: jose.torres@uv.es).

$\mathrm{S}$. Worm is with the Deutsches Elektronen-Synchrotron (DESY), Zeuthen, Germany (e-mail: steven.worm@cern.ch).

Color version of one or more of the figures in this article are available onilne at xxxxxxxxxxx.

Digital Object Identifier xxxxxxxxxxxxxxxxx.
MALTA2 chip, which is part of a development that targets the specifications of the outer pixel layer of the ATLAS Inner Tracker upgrade at the LHC. One of the main challenges for monolithic sensors is a radiation hardness up to $10^{15} 1 \mathrm{MeV} \mathrm{n}$ eq $/ \mathrm{cm}^{2}$ NonIonizing Energy Loss (NIEL) and 80 Mrad Total Ionizing Dose (TID) required for this application. Radiation source and charge injection tests up to $3 \cdot 10^{15} 1 \mathrm{MeV} \mathrm{n}_{\mathrm{eq}} / \mathrm{cm}^{2}$ and $100 \mathrm{Mrad}$ were performed on the MALTA2 sensor and front-end circuit, which still show good performance even after these levels of irradiation, promising for even more demanding applications such as the future experiments at the HL-LHC.

Index Terms-Front-end circuits, Monolithic Active Pixel Sensors, pixel detectors, radiation hardness.

\section{INTRODUCTION}

$\mathbf{M}$ ONOLITHIC Active Pixel Sensors (MAPS) constitute an attractive alternative to the more largely used hybrid pixel sensors for high-energy physics experiments. Their main advantage stems from the integration of the readout electronics and sensor in the same silicon die, avoiding the expensive finepitch bump bonding. MAPS therefore facilitate significantly the detector assembly and reduce the production cost. Without bump bonding, they also tend to offer a smaller pixel pitch and thus a better spatial resolution. Furthermore, the sensor capacitance can be made so small $(<5 \mathrm{fF})$ that it can offer a higher voltage signal even with a reduced sensor thickness, i.e. a lower generated charge. This leads to a better powerperformance ratio, allowing a significant reduction of the material related to the powering and cooling of the detectors. A lower material budget reduces the probability for multiple scattering of the particles emerging from the interaction point, improving the impact parameter resolution and momentum resolution on the reconstructed tracks and the overall detection efficiency of a tracker. Encouraging results from CMOS sensor prototypes [1] prompted the development of large-scale CMOS sensors which would meet the requirements of the outer pixel layer of the ATLAS inner tracker (ITk) upgrade [2]. This entails a NIEL tolerance up to $10^{15} 1 \mathrm{MeV} \mathrm{n}$ eq $/ \mathrm{cm}^{2}$, a TID tolerance up to $80 \mathrm{Mrad}$ and a timing response within $25 \mathrm{~ns}$ with a power density below $500 \mathrm{~mW} / \mathrm{cm}^{2}$. To this end, the $20.6 \mathrm{~mm} \times 20.2 \mathrm{~mm}$ MALTA sensor was designed in the TowerJazz $180 \mathrm{~nm}$ imaging technology. The MALTA pixel matrix features a sensor with a small collection electrode, an open-loop charge-sensitive front-end and a fast, low-power, asynchronous digital readout architecture [3]. Measurements on the chip showed a timing response within the specifications 
[4], however, the efficiency was degraded in the pixel corners already after $10^{14} 1 \mathrm{MeV} \mathrm{n}$ eq $/ \mathrm{cm}^{2}$ [5]. This was addressed in a subsequent small-scale prototype called Mini-MALTA [6] by modifying the sensor to enhance the lateral electric field and improve charge collection. The sensor modifications were then implemented in another large-scale prototype, MALTA2, along with improvements on the front-end circuit. This paper presents the design of the MALTA2 front-end, able to cope with the ATLAS ITk outer pixel layer requirements. For this circuit, the main challenge is to amplify the generated charge with high pixel-to-pixel uniformity and low noise, which are the key features to set low charge thresholds to determine particle hits and obtain good detection efficiencies. Extensive characterization of the prototype is currently in progress and the first measurements, performed on samples irradiated up to $3 \cdot 10^{15} 1 \mathrm{MeV} \mathrm{n}$ eq $/ \mathrm{cm}^{2}$ and $100 \mathrm{Mrad}$, are shown here.

\section{SENSOR}

The cross section of the standard sensor in the TowerJazz $180 \mathrm{~nm}$ CMOS imaging technology is shown in Fig. 1a. It implements a small collection electrode, defined by an n-well implant, which is located inside the sensing volume, typically a high-resistivity p-type epitaxial layer. The in-pixel circuitry is placed outside the collection electrode and it is shielded from it by a deep p-well, which avoids collection of the signal charge by parts of the circuit other than the designated collection electrode. The key features of the technology are therefore the deep p-well, which allows full CMOS in-pixel circuitry, and the possibility to use different starting materials compatible with particle detection. A reverse bias voltage is applied to the substrate to increase the depletion zone within the sensor. To further enhance the depletion around the collection electrode, a reverse bias is typically applied also to the p-well of the NMOS transistors. For visible light, the signal is generated within a depth of a few microns, whereas high energy particles generate charge over the full thickness of the silicon $(\sim 60$ electron/hole pairs per micron traversed [7]) which needs to be collected within the time resolution of the event reconstruction. For the TowerJazz standard sensor of Fig. 1a, the epitaxial layer is only partially depleted and the signal charge generated outside the depletion area is collected by diffusion (with a collection time of $\sim 100 \mathrm{~ns}$ ). The ATLAS experiment has more stringent timing requirements, since the particle hits have to be associated to different bunch crossings, $25 \mathrm{~ns}$ apart from each other. To obtain a faster response, the depletion zone needs to be extended over the whole sensitive layer. The objective is to push the charge carriers towards the collection electrode by drift and thus reduce the collection time. Faster collection times also reduce the probability for charge carriers to get captured by the radiation-induced defects, improving the sensor tolerance to NIEL. To achieve full depletion of the sensor volume, the process has been modified [8] adding a uniform low dose $\mathrm{n}$ - layer under the deep p-well, as shown in Fig. 1b. This creates a planar junction deep in the epitaxial layer and the depletion extends immediately over the full pixel area. The epitaxial layer fully depletes if a sufficiently large reverse bias voltage is applied to the substrate. However,

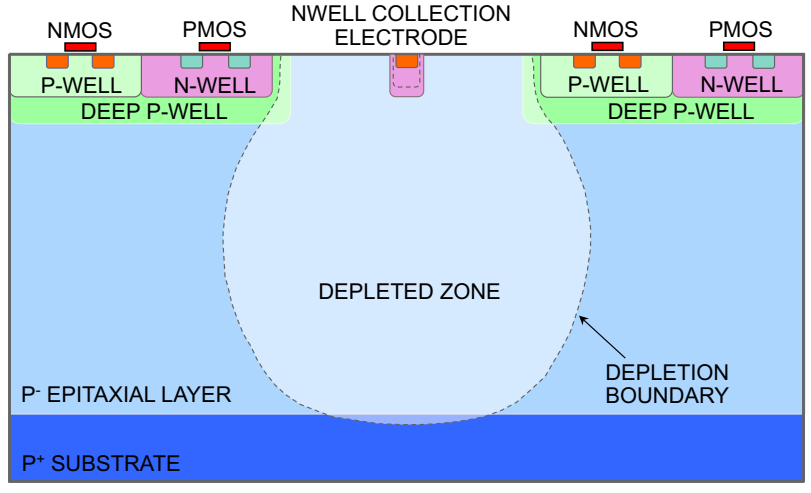

(a)

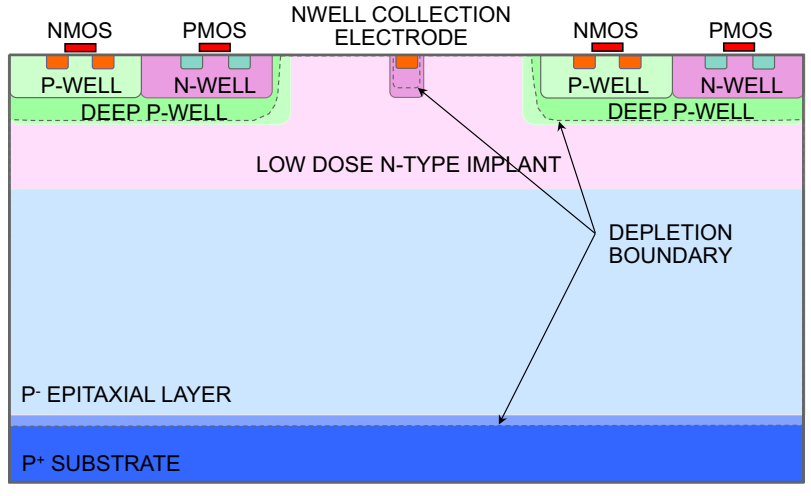

(b)

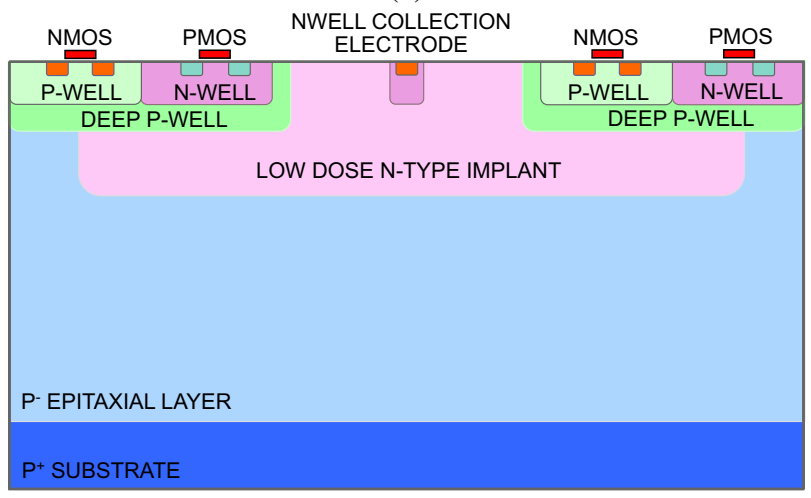

(c)

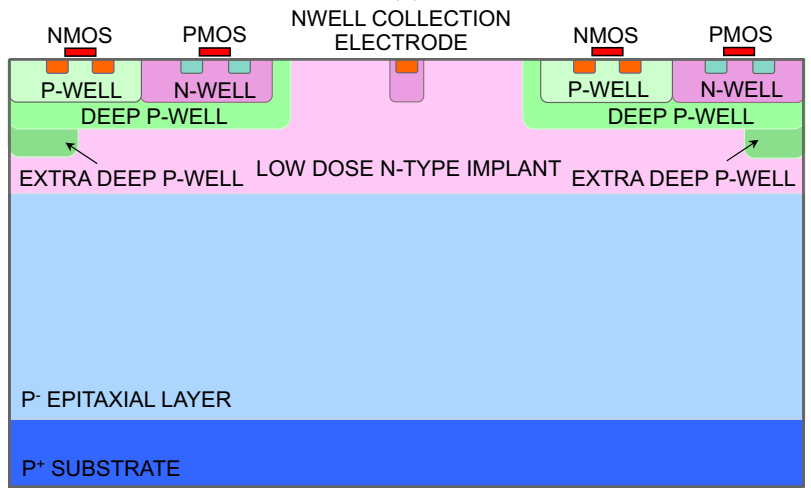

(d)

Fig. 1: Cross section of the TowerJazz $180 \mathrm{~nm}$ CMOS imaging sensor (a) standard process (b) modified process with low dose $\mathrm{n}$ - implant (c) with gap in the low dose $\mathrm{n}-$ implant (d) with extra deep p-well. Sensors in (c) and (d) also fully deplete with a sufficiently large reverse bias. 
despite the full depletion, the lateral electric field in the pixel corners and along the pixel edges is still quite low, resulting in a relatively long collection time and hence a high probability for the charge to get captured by the radiationinduced traps which leads to efficiency loss in these regions [5]. The strength of the lateral electric field in the corner regions can be increased by introducing a lateral gradient in the doping profile [9]. This can easily be achieved by removing the $n-$ layer in the pixel edges, as shown in Fig. 1c, or with the introduction of an extra deep p-well implant, as shown in Fig. 1d [6].

The advantage of the small collection electrode is its low sensor capacitance, key to achieve a low analog power consumption [10]. The signal-to-noise ratio (SNR) of the analog front-end can be calculated comparing the input signal created by the ionization of a high-energy particle to the input-referred noise. The latter is typically dominated by the input transistor thermal noise, inversely proportional to the square root of its transconductance $\mathrm{g}_{\mathrm{m}}$, and can be expressed as an equivalent series voltage. The input signal can be calculated as the ratio of the generated charge divided by the sensor capacitance, i.e. the $\mathrm{Q} / \mathrm{C}$ ratio. Therefore, assuming the front-end power consumption dominated by the input transistor current, one can write

$$
\frac{S}{N} \propto \frac{Q}{C} \sqrt{g_{m}} \propto \frac{Q}{C} \sqrt[m]{P},
$$

with $\mathrm{m}=2$ for the input transistor in weak inversion, where $\mathrm{g}_{\mathrm{m}}$ is proportional to the biasing current, or $\mathrm{m}=4$ in strong inversion, where the $\mathrm{g}_{\mathrm{m}}$ is proportional to the square root of the biasing current. Rearranging, for a fixed SNR and bandwidth

$$
P \propto\left(\frac{Q}{C}\right)^{-m},
$$

with $2 \leq \mathrm{m} \leq 4$, depending on the operating point of the input transistor. The expression states that the required power consumption to reach a given SNR is heavily dependent on the $\mathrm{Q} / \mathrm{C}$ ratio, and it reduces significantly for a smaller sensor capacitance. It is therefore important to mention that the process modification does not introduce any penalty on the sensor capacitance, provided that the doping of the additional $\mathrm{n}$ - layer is sufficiently low for the depletion zone to extend up to the n-well collection electrode [8]. This paper shows results for pixels which implement the sensor from Fig. 1d. The epitaxial layer is $30 \mu \mathrm{m}$ thick and has a resistivity larger than 1 $\mathrm{k} \Omega \cdot \mathrm{cm}$. The collection electrode is an octagonal shaped $\mathrm{n}$-well with a diameter of $2 \mu \mathrm{m}$, distanced $4 \mu \mathrm{m}$ in all the directions from the surrounding p-well containing the circuitry. This geometry is the result of a trade-off between a small sensor capacitance $(<5 \mathrm{fF})$ and a large lateral electric field [11], with collection times in the nanosecond range [9]. The sensor in this modified process was tested up to $10^{15} 1 \mathrm{MeV} \mathrm{n}$ eq $/ \mathrm{cm}^{2}$ [1], still showing good tolerance to NIEL. The transistors also have a good tolerance to TID due to the thin oxide thickness provided by the technology [12].

\section{FRONT-END}

The front-end is a continuously active circuit which performs the reset of the collection electrode, the amplification

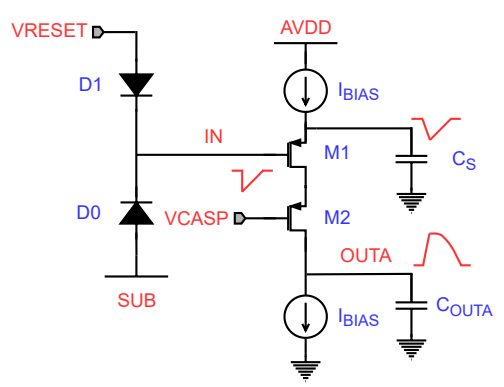

(a)

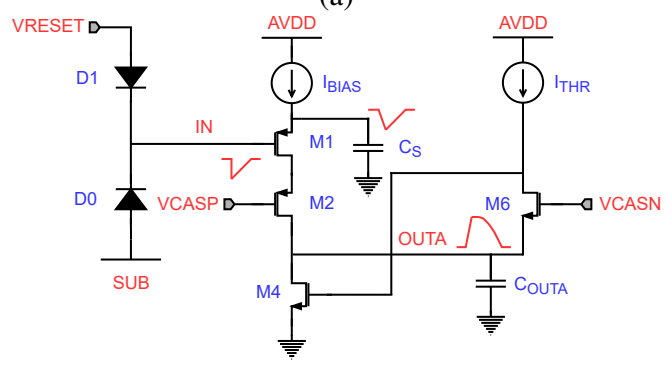

(b)

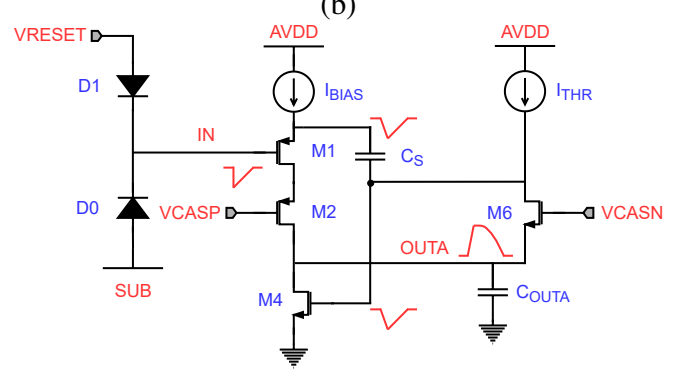

(c)

Fig. 2: Front-end principle: (a) simplified schematic (b) with feedback to adjust the operating point (c) with additional gain mechanism.

of the generated charge, the shaping of the analog signal and the digitization of this signal through a discrimination stage. The basic principle of the amplification stage is illustrated in Fig. 2a. The reset mechanism uses the diode D1 to reset the collection electrode voltage. When no charge is collected by the collection electrode, D1 is biased by the leakage current of the sensor diode D0. Upon a particle crossing, the electrode collects the generated electrons and a negative voltage step with an amplitude of $\Delta \mathrm{V}=\mathrm{Q} / \mathrm{C}$ is generated on it. This causes the reset diode to conduct more current and to slowly charge the input node back up to its original value, which can take several hundreds of $\mu \mathrm{s}$. The reset diode is implemented with a small $\mathrm{p}+$ implant in the $\mathrm{n}$-well of the collection electrode, adding only a small capacitance to the sensor. A chargesensitive amplifier which integrates the generated charge in a feedback capacitor [13] is typically used in these applications. The small sensor capacitance (large $\mathrm{Q} / \mathrm{C}$ ) opens the possibility to integrate the charge on the sensor itself and use an openloop amplifier, more compact and power-efficient. The input node (gate of the transistor M1) is connected directly to the collection electrode. The input transistor M1 acts as a source follower and, when the input voltage drops because of the collected charge, forces its source to follow transferring charge 
from the capacitance $\mathrm{C}_{\mathrm{S}}$ to the output node capacitance $\mathrm{C}_{\text {OUTA }}$. Ideally, for the voltage on OUTA, one can write

$$
\Delta V_{\text {OUTA }}=\frac{Q_{S}}{C_{\text {OUTA }}}=\frac{C_{S} \cdot \Delta V_{I N}}{C_{\text {OUTA }}}=\frac{C_{S}}{C_{\text {OUTA }}} \frac{Q_{I N}}{C_{I N}} .
$$

Therefore, a large gain is obtained for $\mathrm{C}_{\mathrm{S}}>>\mathrm{C}_{\mathrm{OUTA}}$. The overall effective sensor capacitance is the sum of the sensor junction capacitance, the reset diode parasitic capacitance, the input line and the input transistor gate capacitance. After settling, the following action of the input transistor reduces the contribution of its gate-source capacitance to the total capacitive load on the electrode. Furthermore, the cascode transistor M2 mitigates the Miller effect on the gate-drain capacitance of the input transistor. A more practical implementation of the circuit is shown in Fig. 2b. Since the two I IIAS current sources are difficult to match, a low-frequency feedback which sets the operating point of the transistor M4 is introduced: its gate voltage is now adjusted for it to sink $\mathrm{I}_{\mathrm{BIAS}}+\mathrm{I}_{\mathrm{THR}}$, where $\mathrm{I}_{\mathrm{THR}}$ is a small fraction of the main biasing current $\mathrm{I}_{\text {BIAS. }}$. This branch defines the DC voltage of the amplifier output node and its return to baseline. Upon a particle hit, when the voltage on OUTA rises, the gate-source voltage of the transistor M6 reduces, forcing $\mathrm{I}_{\mathrm{THR}}$ to charge up the gate of the transistor M4, discharging OUTA and bringing it back to its baseline value. An additional gain mechanism is introduced by connecting the capacitance $\mathrm{C}_{\mathrm{S}}$ to the gate of the transistor M4, as done in Fig. 2c. A part of the signal on the input transistor source is now transferred to the gate of the transistor M4, which behaves as a common-source device. In this scheme, the capacitance $\mathrm{C}_{\mathrm{S}}$ plays an important role not only for the gain of the amplifier but also in determining its return to baseline, since it is connected to the feedback node, i.e. the gate of the transistor M4. A larger $\mathrm{I}_{\mathrm{THR}}$ increases the speed of the feedback loop, resulting in a faster return to baseline, but could also provide an excessive filtering at low frequencies on the gate of the transistor M4, reducing the amplifier gain. Indeed, due to this low-frequency internal feedback and the gain mechanism introduced by the capacitance $\mathrm{C}_{\mathrm{S}}$, the front-end response behaves at low frequencies as a high-pass filter. The gain, however, drops at high frequencies due to the poles on the output and feedback node which exhibit a large impedance. Overall, the circuit is characterized by a bandpass response and no additional shaping is required after the amplification stage. The bandwidth of the amplifier can be optimized for the signal bandwidth to improve the SNR or, in other words, reduce the Equivalent Noise Charge (ENC).

The complete front-end circuit which includes the amplifier and discriminator is shown in Fig. 3. The capacitance $\mathrm{C}_{\mathrm{S}}$ is implemented with a PMOS device whose source, bulk and drain are connected together to exploit the capacitance of the MOS structure in inversion. The capacitor $\mathrm{C}_{\text {OUTA }}$ includes only the parasitic contributions of transistors connecting to it, since it needs to be as low as possible. The input transistor M1 is placed together with the capacitor $\mathrm{C}_{S}$ in a separate nwell connected to its source to eliminate the body effect and achieve a gain close to unity for the input source follower. An improvement to the circuit from Fig. 2c is provided by cascoding the transistor M4. For good timing performance, a

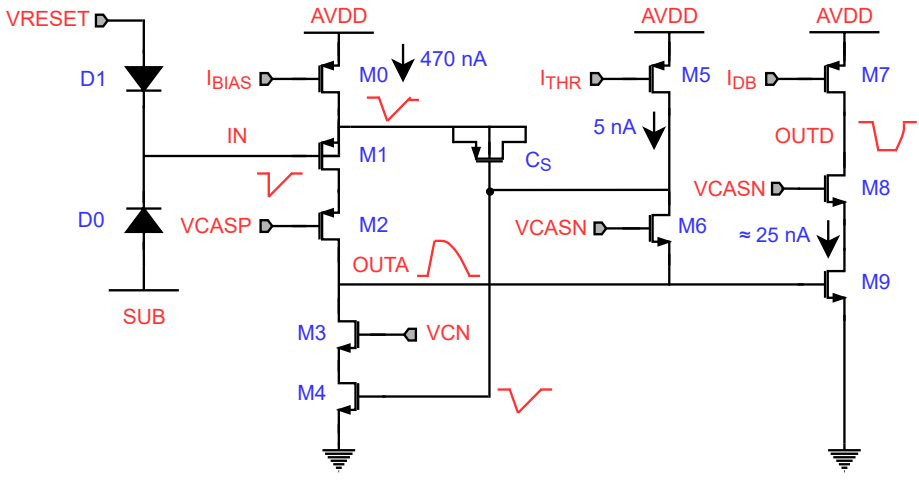

Fig. 3: Complete front-end schematic with discriminator.

large transconductance is required for this transistor. However, a too large aspect ratio would increase the output parasitic capacitance, detrimental both for gain and speed itself. The cascode decouples the transistor M4 from the output node, giving more freedom in its sizing, and is optimized for a reduced output capacitance. Additionally, it increases the output impedance of the amplifier, which is thus dominated by the transconductance of the transistor M6 which works in weak inversion, leading to a higher gain. The discriminator consists of a common-source amplification stage, the transistors M7M9, which can be better seen as a current comparator. In steady state, the output baseline of the amplifier sets the standby current of the transistor M9, while the transistor M7 is biased to provide a current $\mathrm{I}_{\mathrm{DB}}$ higher than the DC current forced by the transistor M9, charging the node OUTD to the supply voltage. As the signal on OUTA rises upon a particle hit, the current drawn by the transistor M9 increases, eventually exceeding $\mathrm{I}_{\mathrm{DB}}$ and discharging the output node to ground. The threshold of the discriminator is therefore controlled by the $\mathrm{I}_{\mathrm{DB}}$ current setting and the amplifier output baseline (through VCASN). The cascode transistor M8 is again used to reduce the large capacitance penalty on OUTA due to the Miller effect on the transistor M9 and the coupling between this node and the rail-to-rail OUTD signal. In the actual frontend implementation, three parallel NMOS switches are placed between the source of the transistor M9 and the ground. These switches are controlled by three different digital signals which are connected to all the pixels in a row, column or diagonal. If all the switches are open, the discriminator is disabled and cannot generate an output pulse. This logic gives the option to address a pixel and disable it in case it generates an excessive noise hit rate.

The circuit is designed to have peaking times in the order of tens of ns with a low power consumption. The amplifier peaking time is defined by $g_{m} / C_{\text {OUTA }}$ where $g_{m}$ is the transconductance of the amplifying devices and COUTA the load capacitance. The transistors' dimensions and the layout are therefore optimized to reduce the load capacitance $\mathrm{C}_{\text {OUTA }}$ to less than $\sim 5 \mathrm{fF}$. The main biasing current $\mathrm{I}_{\text {BIAS }}$ needs to be $\sim 470 \mathrm{nA}$ to reach the target timing response. The $\mathrm{I}_{\mathrm{THR}}$ current, typically a few nA, and the discriminator off current, typically a few tens of nA, need to be added to the IBIAS current to obtain the total current consumption which is $\sim 500 \mathrm{nA}$. 
With a supply voltage of $1.8 \mathrm{~V}$, the total power consumption is less than $1 \mu \mathrm{W}$ per pixel, which is used efficiently thanks to the current reuse between the input follower M1 and the common-source device M4. A parasitic-extracted simulation of the transient waveforms at the input IN, analog output OUTA and discriminator output OUTD of the front-end with the charge threshold set to $100 \mathrm{e}^{-}$is shown in Fig. 4. The solid lines show the response for a collected charge of 250 $\mathrm{e}^{-}$, the dashed lines for a charge of $1000 \mathrm{e}^{-}$. The simulation was performed using a current pulse at the input, i.e. by injecting the input charge uniformly in a collection time of $1 \mathrm{~ns}$. The sensor is modelled as a capacitance of $2.5 \mathrm{fF}$, which is a value previously measured on prototype chips [14], in parallel with a leakage current source of $10 \mathrm{pA}$. The red curves represent the input signals and show that the voltage step on the electrode is proportional to the collected charge. The blue curves represent the amplified signals on OUTA. The front-end gain is non-linear since the transistor M6 dynamically turns off as the output voltage rises, offering a larger impedance on the output node. At threshold, the gain is $\approx 1.9 \mathrm{mV} / \mathrm{e}^{-}$, whereas for a charge of $250 \mathrm{e}^{-}$, as seen in Fig. 4, it is $\approx 2.5 \mathrm{mV} / \mathrm{e}^{-}$. For larger charges, the analog output signal on OUTA is sufficiently large to push the cascode transistor M2 out of saturation and the front-end gain drops. This makes the cascode ineffective, so the equivalent input capacitance increases due to the Miller effect. The input signal is therefore lower during this transition time, as shown from the dashed red curve of Fig. 4, and a saturation of the analog output signal is reached. However, its Time over Threshold (ToT), i.e. the duration of the discriminator output pulse shown in green in Fig. 4, increases close to linearly with the input charge. Indeed, the ToT depends on the time required for the feedback circuit to charge up the capacitor $\mathrm{C}_{\mathrm{S}}$ through the current $\mathrm{I}_{\mathrm{THR}}$.

ङ

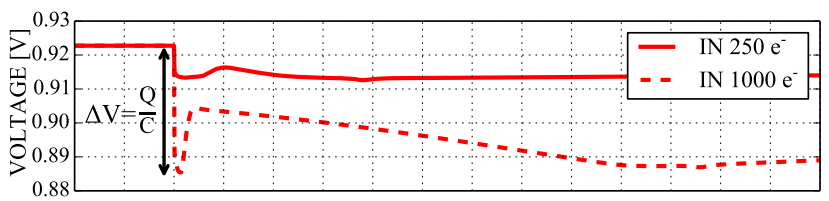

อ
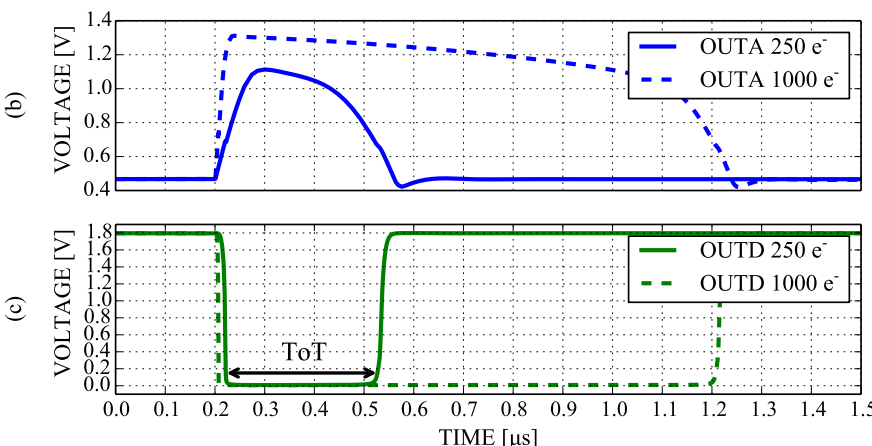

Fig. 4: Front-end simulated transient response with a threshold of $100 \mathrm{e}^{-}$: (a) signals at the sensing node, (b) signals at the output of the amplifier, (c) signals at the output of the discriminator.

For a reliable operation of the sensor, the front-end ENC has to be considerably lower than the charge threshold. Even though the circuit is highly non-linear, a small-signal AC noise analysis helps to gain insights about the main noise sources. The results of this type of simulation are shown in the Table I which reports the highest contributions with the corresponding percentage of the output noise, integrated from $1 \mathrm{~Hz}$ to 10 $\mathrm{GHz}$, i.e. in a frequency range much broader than the amplifier passband, which extends from $\sim 15 \mathrm{kHz}$ to $\sim 10 \mathrm{MHz}$.

TABLE I: Output noise contributions

\begin{tabular}{|c|c|c|}
\hline Device & Noise source & Percentage \\
\hline M1 & Thermal noise & $53 \%$ \\
\hline M4 & Thermal noise & $36 \%$ \\
\hline D1 & Shot noise & $2.5 \%$ \\
\hline M6 & Thermal noise & $2 \%$ \\
\hline M5 & Thermal noise & $1.75 \%$ \\
\hline
\end{tabular}

The thermal noise of the amplifying devices M1 and M4 is the dominating noise contributor, as assumed in the previous section for the derivation of the power consumption as a function of the sensor capacitance. The next main contributor is the shot noise from the reset diode. The simulation was performed with a leakage current of $10 \mathrm{pA}$ which is an overestimate for an unirradiated sensor. A small percentage of noise comes from the $\mathrm{I}_{\mathrm{THR}}$ current source and the device M6 which define the baseline of the amplifier output. Other contributions to the output noise are much less significant. An important noise source not accounted for in the simulation is the Random Telegraph Signal (RTS) noise. Indeed, the simulation models do not include RTS noise which is therefore difficult to estimate during design. The most critical devices for RTS noise are again the transistors M1 and M4 whose sizing required an iterative process [6]. Increasing the gate area of the transistor M1 to combat RTS noise results in a larger effective sensor capacitance. A gate area of 0.18 $\mu \mathrm{m}^{2}$ has been chosen since it is a good compromise between capacitance penalty and noise. A gate area of $\sim 2.4 \mu \mathrm{m}^{2}$ has instead been chosen for the transistor M4 since it exhibits a larger noise transfer function to the output node and the RTS noise is typically larger in NMOS transistors. One of the main parameters for good noise performance is the size of the capacitance $\mathrm{C}_{\mathrm{S}}$ : a larger capacitance provides dynamically more charge to the output node for the same input signal and improves the coupling between the input transistor source and the gate of the transistor M4. From a frequency standpoint, it widens the amplifier passband towards lower frequencies where the input signal has a large frequency content. The output signal therefore increases more than the noise level and a larger SNR is obtained. The PMOS transistor implementing this capacitor has a gate area of $\sim 14.24 \mu \mathrm{m}^{2}$, providing a capacitance of $\sim 114 \mathrm{fF}$, and it is one of the largest components of the circuit.

Apart from the noise, another limit to the minimum operating threshold is the pixel-to-pixel variation of the transistor parameters which causes the threshold to vary over the matrix. It is well known that the transistors' mismatch scales down with the square root of their area [15]. The pixel size is however limited and often dictated by the target sensor spatial resolution. To optimize the space, it is therefore necessary to identify the devices with the largest impact on the threshold 
dispersion and increase their area. In the amplification stage, the most critical devices are the transistors M5 and M6. As previously said, the current $\mathrm{I}_{\mathrm{THR}}$ of the transistor M5 defines the speed of the feedback loop and significantly influences the amplifier gain. Regarding the transistor M6, its gate-source voltage directly defines the amplifier output baseline, setting the discriminator DC current and hence its switching threshold. The transistor M5 is biased with a current of only a few nA and therefore operates in weak inversion, which makes the impact of its mismatch even more prominent. For these reasons, it is designed with a low aspect ratio and a large area $\left(20 \mu \mathrm{m}^{2}\right)$. The size of the transistor M6, however, cannot be increased to the same extent because of the capacitance penalty on the output node. In the discriminator stage, the input transistor M9 is the main critical device: a variation of its threshold voltage directly shifts the switching point of the discriminator, appearing effectively as an offset. As for the transistor M6, it has to be kept small to prevent increasing the amplifier output capacitance and it represents the largest contribution (nearly $50 \%$ ) to the overall threshold dispersion. Fig. 5 shows the front-end probability to generate a hit as a function of the input charge, obtained with transient-noise simulations (Fig. 5a) and Monte Carlo simulations for transistors' mismatch (Fig. 5b). The mean value of the Gaussian error function fit gives the nominal threshold, which is $\sim 100 \mathrm{e}^{-}$, whereas its standard deviation gives the ENC and the pixel-to-pixel threshold variation for the two simulations respectively, which are $\sim 6.4$ $\mathrm{e}^{-}$and $\sim 2.5 \mathrm{e}^{-}$.

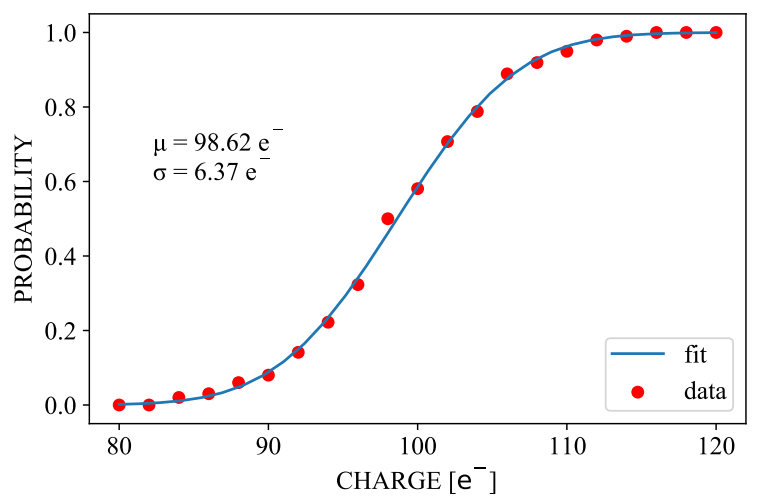

(a)

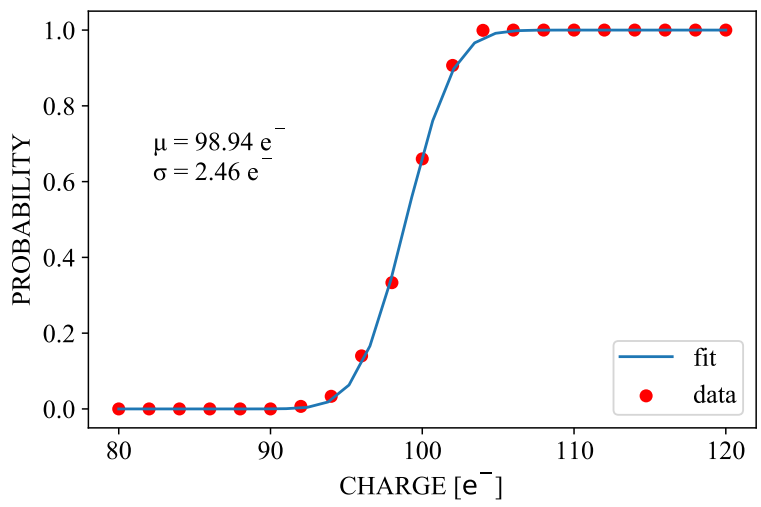

(b)

Fig. 5: Hit probability as a function of the input charge with simulated (a) transient noise and (b) transistors' mismatch.
The radiation effects that influence the front-end operation and performance include the increase of the sensor leakage current due to NIEL and the TID effects that affect the transistor characteristics. For a good tolerance to NIEL, the front-end has been designed to cope with a wide range of sensor leakage currents. As for the tolerance to TID, minimum dimensions have been avoided for the critical devices to mitigate Radiation-Induced Narrow Channel (RINCE) and Short Channel (RISCE) effects [16]. Leakage currents in the order of a hundred pA have been measured for NMOS transistors in this technology after $20 \mathrm{Mrad}$ of TID [17]. Since the $\mathrm{I}_{\mathrm{THR}}$ current can be below $1 \mathrm{nA}$, the transistor M6 has been designed as an enclosed layout transistor (ELT [18]) and is surrounded by a $\mathrm{p}+$ guard ring to prevent any leakage to neighboring devices. These precautions double its area but they are necessary to ensure radiation hardness. The layout of the pixel is shown in Fig. 6. The $2 \mu \mathrm{m}$ octagonal collection electrode, distanced $4 \mu \mathrm{m}$ from the surrounding $\mathrm{p}$-well of the circuitry, is placed in the centre of the pixel. The front-end circuit occupies an area of $\sim 160 \mu \mathrm{m}^{2}$ and is placed to the left of the collection electrode with other analog circuitry. The latter includes decoupling capacitors and a testing circuit which can capacitively inject a tuneable amount of charge to the collection electrode. The rest of the pixel is occupied by the digital readout circuitry for a total pixel area of $36.4 \mu \mathrm{m} \times$ $36.4 \mu \mathrm{m}$, leading to an analog power density of $75 \mathrm{~mW} / \mathrm{cm}^{2}$ over the matrix.

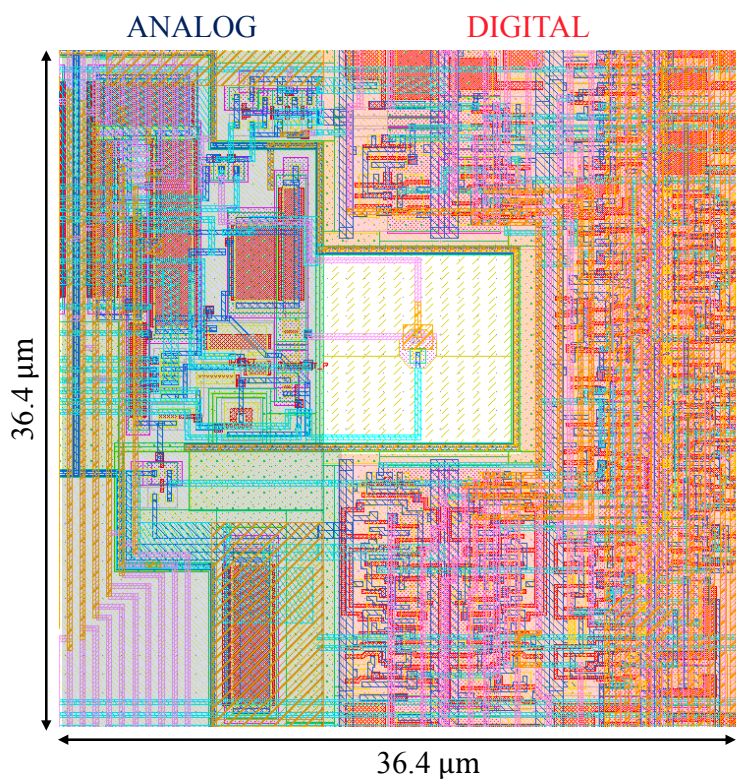

Fig. 6: Layout of the pixel.

The MALTA2 chip has a size of $10.12 \mathrm{~mm} \times 20.2 \mathrm{~mm}$ and integrates a matrix of $224 \times 512$ pixels. It features an asynchronous readout which avoids propagation of the clock in the matrix to reduce the digital power consumption. Upon a particle hit, the in-pixel digital circuitry sends a pattern of short pulses corresponding to the pixel address to the periphery on a digital data bus. The pixels are organized in double columns, where each double column has a dedicated bus for the transmission of the data down to the periphery. Here, a binary 
tree-like structure which merges the hits of the whole matrix onto a single bus is implemented. In case of simultaneous events, this logic delays one of the two hits in time while keeping track of the delay for later reconstruction. The final word is 40 bits wide and is transmitted off chip via LVDS drivers, which are designed to operate at a maximum speed of 5 Gbps [19], sufficiently high for the pixel detector to cope with the ATLAS ITk outer layer hit rate of $100 \mathrm{MHz} / \mathrm{cm}^{2}$. A micrograph of the MALTA2 chip is shown in Fig. 7.

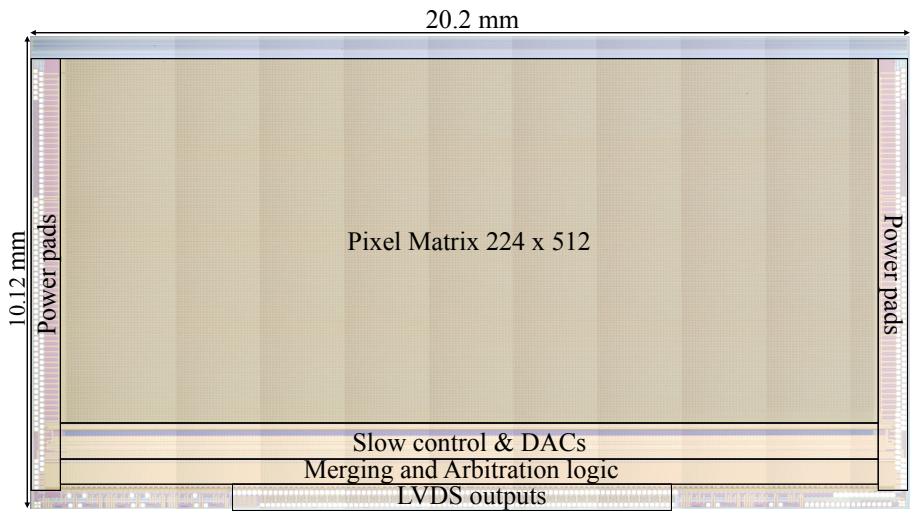

Fig. 7: Micrograph of the MALTA2 chip.

\section{FRONT-END CHARACTERIZATION}

To test the performance of the front-end, a special set of pixels which allows the monitoring of its analog output has been included on the left and right side of the matrix. In these pixels, the front-end analog output is buffered to an output pad with a two-stage source follower with a gain close to 1 . The first stage is optimized to match closely the discriminator input capacitance to have the same amplifier output load as in the other pixels of the matrix. An oscilloscope is used to monitor the output pad through a low-capacitance active probe and the full buffering system is designed not to degrade the signal timing. The front-end speed can be evaluated with the plot of Fig. 8 which shows the time walk curve, i.e. the time for the amplifier output to reach the discriminator threshold as a function of the charge. The conversion between charge and amplitude is derived through the charge injection circuitry. For this measurement, the front-end operates with the nominal bias settings ( $\sim 1 \mu \mathrm{W}$ power consumption) and the oscilloscope is set to trigger with a signal of $\sim 100 \mathrm{e}^{-}$. The waveforms are collected while exposing the chip to a ${ }^{90} \mathrm{Sr}$ radioactive source which undergoes $\beta^{-}$decay emitting electrons that generate an ionization signal close to a Minimum Ionising Particle (MIP). The most probable value of charge deposition for a MIP in the $30 \mu \mathrm{m}$ thick epitaxial layer is $\sim 1800 \mathrm{e}^{-}$[7]. The signal is collected by a cluster up to 4 pixels and the seed pixel, the one with the largest signal, has a charge $\geq 1 / 4$ of a MIP charge. Events with high charges $\left(\gtrsim 1200 \mathrm{e}^{-}\right)$arrive with a time walk close to the minimum value of $\sim 10 \mathrm{~ns}$. With respect to the ATLAS application, an event is considered in time when it falls within $25 \mathrm{~ns}$ from the lowest possible time walk. As can be noticed in Fig. 8, the in-time threshold corresponds to an input charge of $\sim 200 \mathrm{e}^{-}$. Less than $10 \%$ of the hits are below the in-time threshold. Statistically, these are mostly caused by non-seed pixels, with a neighboring seed pixel which is likely to collect a charge above the in-time threshold.

The front-end timing can also be studied through the matrix digital readout. An increasing amount of charge can be injected in a specific pixel with the aforementioned charge injection circuitry. The time of arrival of the generated hits can then be compared to a time reference. This procedure has been performed using as time reference the charge injection trigger pulse sent to the chip. In order to do so, this signal is also sent to a 3 ps binning TDC [20] together with a fast-OR signal from the chip. The mean difference between these two signals' time of arrival provides the same time walk curve of Fig. 8. This methodology, however, allows to better study the frontend jitter by evaluating instead the RMS difference of the two signals' time of arrival, which is plotted as a function of the charge in Fig. 9. For each injected charge, ten thousand events are acquired. The time jitter of the reference pulse has been estimated to be below 100 ps, therefore, the values in Fig. 9 are dominated by the front-end jitter which reduces from 4.7 ns at threshold, down to $0.16 \mathrm{~ns}$ for very high input charges.

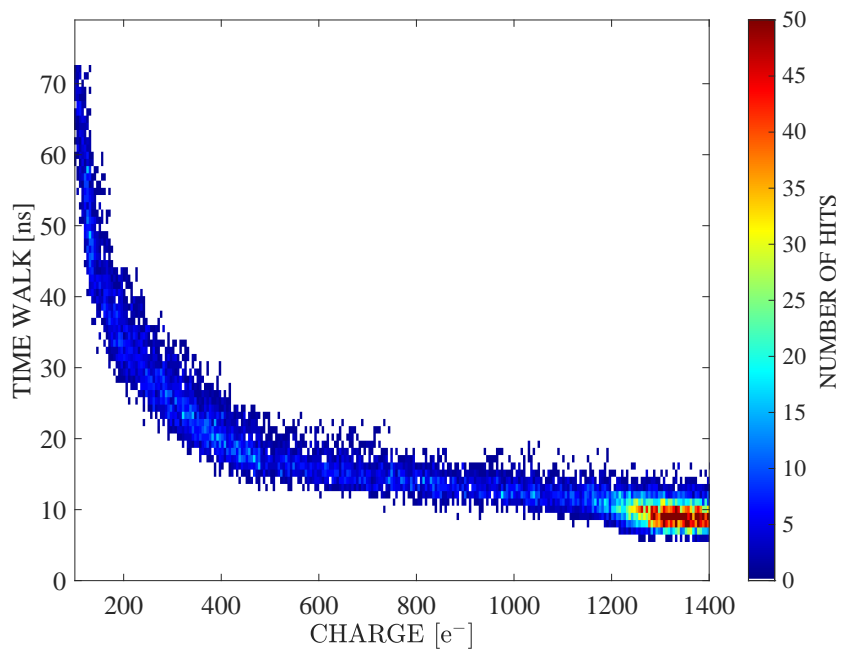

Fig. 8: Time walk curve obtained with a ${ }^{90} \mathrm{Sr}$ source.

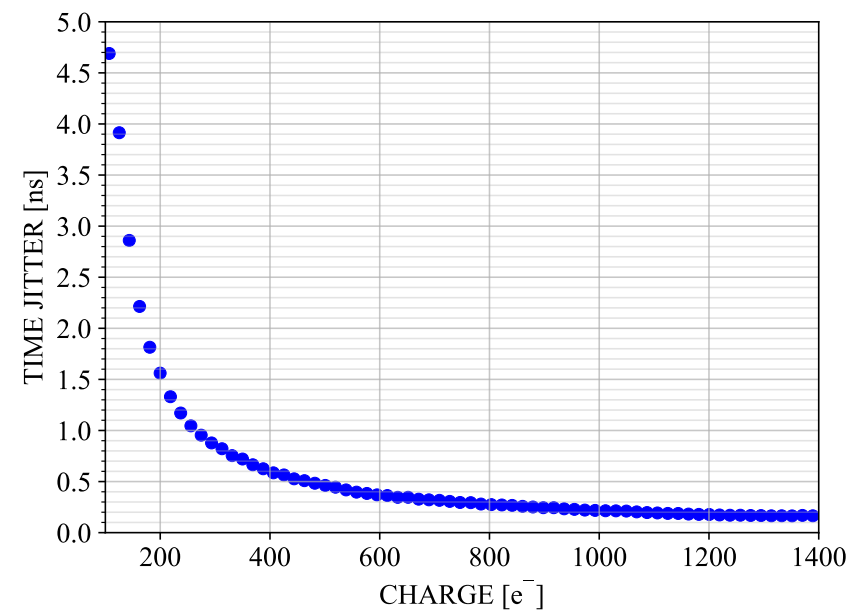

Fig. 9: Dependence of front-end time jitter on charge. 


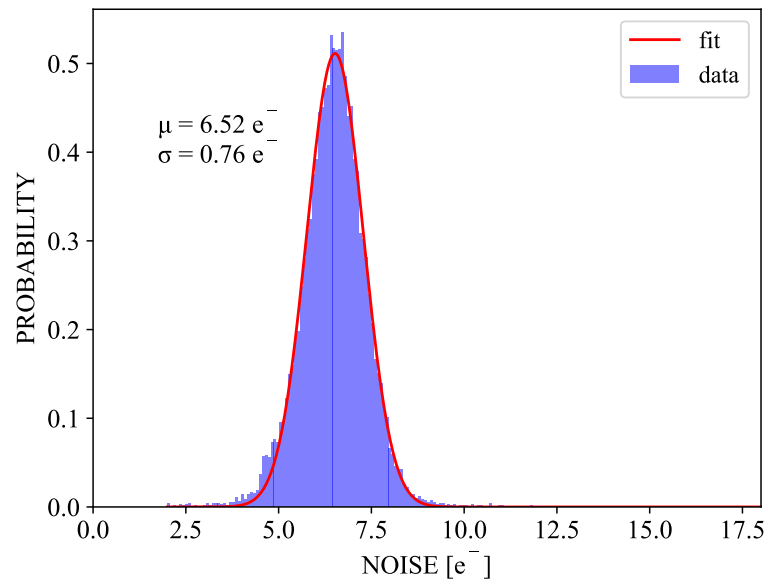

(a)

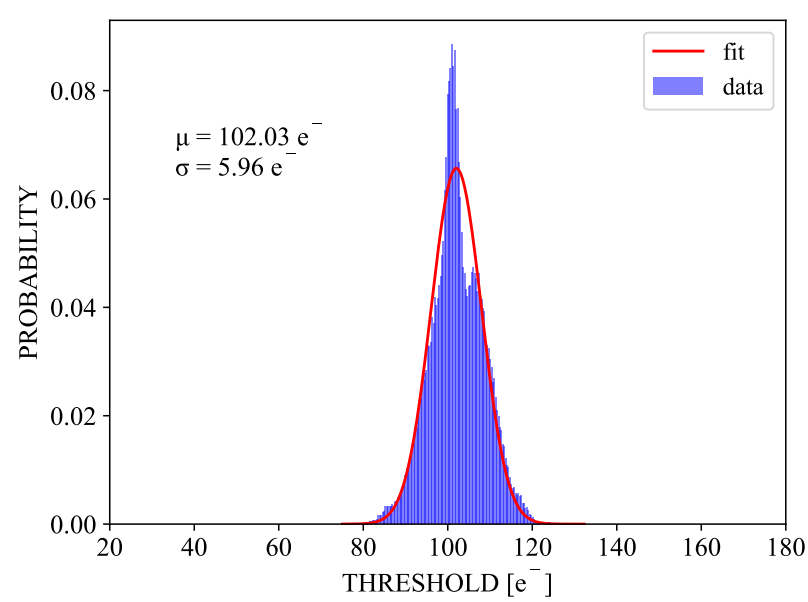

(b)

Fig. 10: Distribution of (a) ENC (b) threshold.

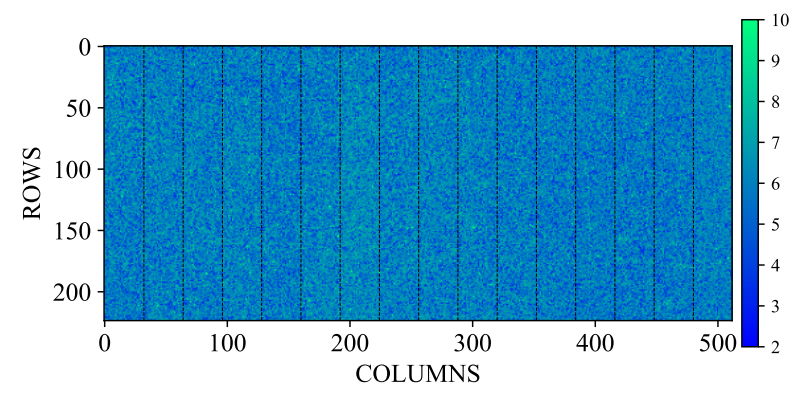

(a)

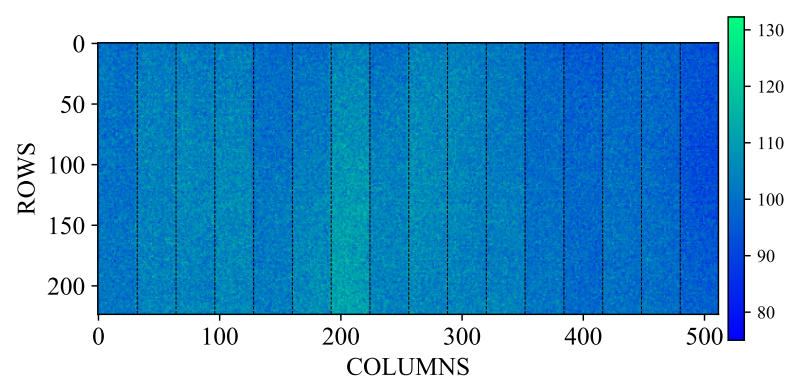

(b)

Fig. 11: 2D map for (a) ENC (b) threshold.

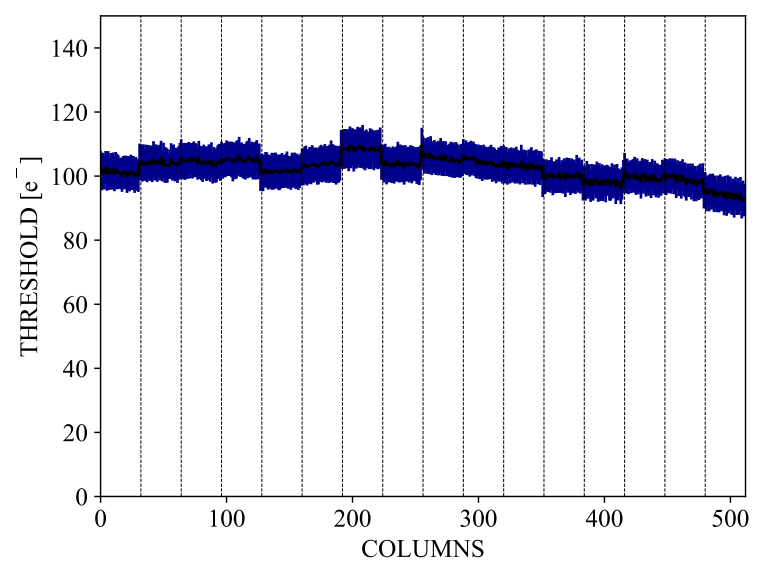

(a)

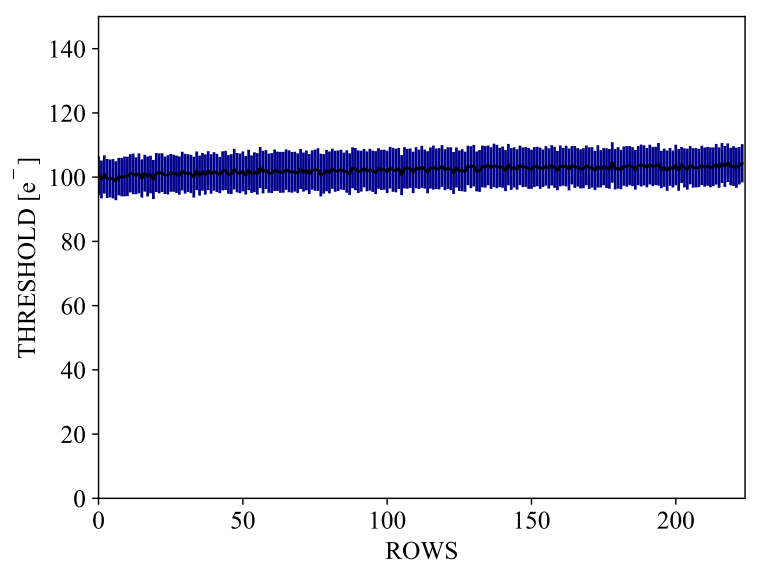

(b)

Fig. 12: Distribution of the threshold vs the (a) columns and (b) rows.

The charge injection circuitry also allows to extract information such as threshold and noise for all the pixels. Fig. 10 shows the threshold and noise distribution for an entire matrix with nominal front-end settings. The average threshold is $\sim 100 \mathrm{e}^{-}$with a variation of $\sim 6 \mathrm{e}^{-}$, more than a factor of 2 higher than the simulated value shown in Fig. 5. The noise distribution has an average of $6.5 \mathrm{e}^{-}$ with a low spread, matching fairly well the simulations. 2D maps of the pixels' threshold and noise are shown in Fig. 11. No systematic effects are observed for the noise. As for the threshold, it is possible to notice a variation of its average over different vertical sections of the matrix. This effect strongly correlates with the scheme of the front-end biasing which is adjusted through DACs in the bottom periphery. The power pads are distributed only along the left and right side of the matrix. For this reason, a horizontal power voltage drop is inevitably present and is estimated to reach $\sim 12 \mathrm{mV}$ in the middle of the matrix. To compensate this effect and avoid a systematic threshold gradient, the biasing DACs have a dedicated mirroring stage for every 32 columns of the matrix 
which shares their local power supply. To better study the threshold behaviour, the distribution of the threshold along the columns with one RMS error bar is shown in Fig. 12a and here the threshold average variation at every biasing group is more clearly visible. A straightforward solution to increase the biasing transistors' area and mitigate this effect is to connect more mirroring stages together, trading-off with the power voltage drop compensation accuracy. This is envisaged for a future prototype. Fig. 12b illustrates the distribution of the threshold along the rows showing a slight vertical gradient which is caused by the mirroring stages at the matrix bottom which load the matrix power grid and introduce a vertical power voltage drop. Considering only pixels within the same biasing group and correcting the systematic vertical gradient, the threshold variation is $\sim 5.1 \mathrm{e}^{-}$. The variation of the NMOS transistors' output conductance with a high reverse bias to the bulk (beyond the normal supply voltage) is not fully covered by the simulation models and this is thought to be the cause of the discrepancy between the simulated and measured threshold variation. Even with a larger pixel-to-pixel mismatch, the chip can be operated reliably with thresholds of $\sim 100 \mathrm{e}^{-}$.

A number of chips have been irradiated with neutrons at the TRIGA reactor in Ljubljana [21] up to $3 \cdot 10^{15} 1 \mathrm{MeV} \mathrm{n}$ eq $/ \mathrm{cm}^{2}$ of NIEL fluence. The chips also received a background TID of $1 \mathrm{Mrad}$ for every $10^{15} 1 \mathrm{MeV} \mathrm{n} \mathrm{n}_{\mathrm{eq}} / \mathrm{cm}^{2}$. During irradiation, the chips were not powered. After irradiation, the chips are stored at a low temperature (below $-20{ }^{\circ} \mathrm{C}$ ) to avoid annealing of the radiation damage. For the same reason, all the measurements of irradiated samples are performed at $-20{ }^{\circ} \mathrm{C}$ which also helps to contain the increase of the sensor leakage current. The chip still shows full functionality after these levels of irradiation.

The effect of the irradiation on the sensor and front-end performance can be studied by evaluating their response to a ${ }^{55} \mathrm{Fe}$ source. The ${ }^{55} \mathrm{Fe}$ isotope decays by emitting X-rays of two characteristic energy peaks: a $\mathrm{K}_{\alpha}$ peak at $5.9 \mathrm{keV}$ and a $\mathrm{K}_{\beta}$ peak at $6.49 \mathrm{keV}$ with a lower probability than for the $\mathrm{K}_{\alpha}$ emission. These $\mathrm{X}$-rays deposit respectively a localized charge of $1639 \mathrm{e}^{-}$and $1803 \mathrm{e}^{-}$. The ${ }^{55} \mathrm{Fe}$ spectra, measured by probing the output pad of a monitoring pixel of an unirradiated sample and a sample irradiated at $3 \cdot 10^{15} 1$ $\mathrm{MeV} \mathrm{n} \mathrm{n}_{\mathrm{eq}} / \mathrm{cm}^{2}$ and $3 \mathrm{Mrad}$, are shown in Fig. 13. Both spectra are obtained with the same front-end settings and number of events. For the unirradiated sample, the $\mathrm{K}_{\alpha}$ and $\mathrm{K}_{\beta}$ peak are visible, whereas this is no longer true for the irradiated sample due to the larger noise. The events below the $\mathrm{K}_{\alpha}$ peak are a result of charge sharing. The ${ }^{55} \mathrm{Fe}$ spectrum provides valuable information about the front-end performance in terms of noise and energy resolution. For this measurement, the amplifier gain has been set as low as possible to prevent output saturation. Given the amplitude of the $\mathrm{K}_{\alpha}$ peak, the front-end gain for the unirradiated sample is estimated to be approximately 0.225 $\mathrm{mV} / \mathrm{e}^{-}$. The width of the $\mathrm{K}_{\alpha}$ peak instead indicates, after correcting for the Fano factor to eliminate the impact of the fluctuation on the generated charge [22], an RMS noise of $\sim 6.8 \mathrm{e}^{-}$which corresponds to an energy resolution of $\sim 57.6$ eV FWHM. The amplitude of the $\mathrm{K}_{\alpha}$ peak is shifted to higher values for the irradiated sample, going from $368.2 \mathrm{mV}$ to $455.6 \mathrm{mV}$. The cause of this $\sim 23.7 \%$ increase is most likely

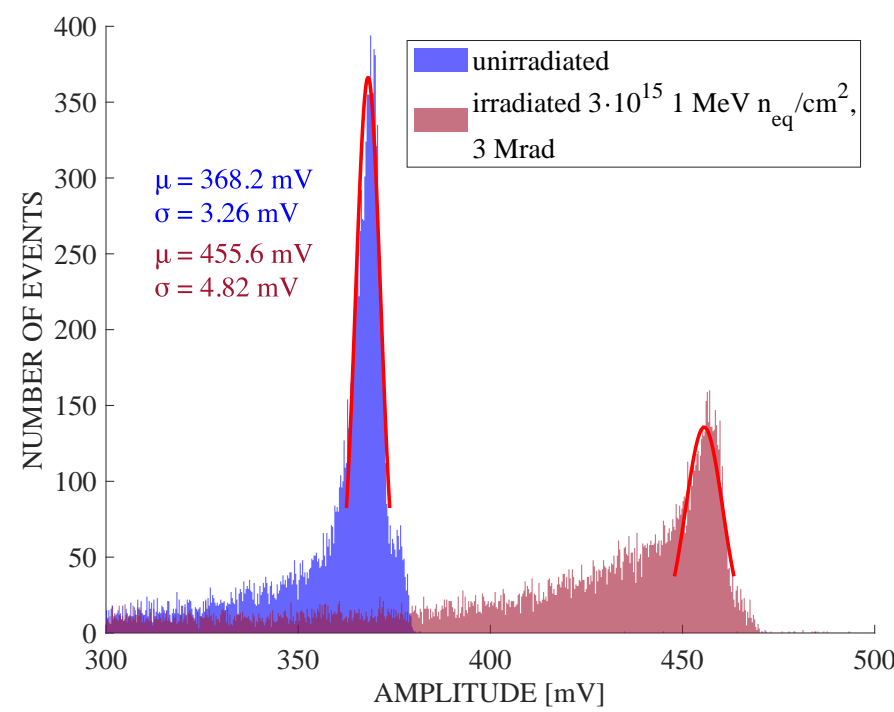

Fig. 13: ${ }^{55} \mathrm{Fe}$ source spectrum for a unirradiated sample and a sample irradiated at $3 \cdot 10^{15} 1 \mathrm{MeV} \mathrm{n} \mathrm{n}_{\mathrm{eq}} / \mathrm{cm}^{2}$ and $3 \mathrm{Mrad}$.

related to the decrease of the effective doping in the $n$ - layer due to NIEL which results in a lower collection electrode capacitance and automatically in a higher voltage signal for the same collected charge at the input of the front-end amplifier. The RMS of the $\mathrm{K}_{\alpha}$ peak however degrades due to the larger sensor leakage and TID-induced noise, giving an RMS noise of $\sim 11.7 \mathrm{e}^{-}$and an energy resolution of $\sim 99.2 \mathrm{eV} \mathrm{FWHM.}$ The higher noise values than the ones shown later in Fig. 14 for samples with the same level of irradiation at $-20{ }^{\circ} \mathrm{C}$ are caused by the sub-optimal front-end operating conditions and the additional noise added by the buffering stages and the measurement setup.

Charge injection tests have also been performed on samples irradiated up to $3 \cdot 10^{15} 1 \mathrm{MeV} \mathrm{n}$ eq $/ \mathrm{cm}^{2}$ and $3 \mathrm{Mrad}$, with a step of $10^{15} 1 \mathrm{MeV} \mathrm{n} \mathrm{n}_{\mathrm{eq}} / \mathrm{cm}^{2}$ and $1 \mathrm{Mrad}$. For a fair comparison, the measurement of the unirradiated sample has been repeated at $-20{ }^{\circ} \mathrm{C}$ and the $\mathrm{I}_{\mathrm{THR}}$ current setting of the front-end has been adjusted to obtain similar thresholds in all the cases. An increasing level of ENC and threshold dispersion as a function of the irradiation level has been noticed. The distribution of ENC and threshold dispersion for an unirradiated sample and a sample irradiated to $3 \cdot 10^{15} 1 \mathrm{MeV} \mathrm{n}$ eq $/ \mathrm{cm}^{2}$ and $3 \mathrm{Mrad}$ are shown in Fig. 14 for reference. The noise average for the unirradiated sample is $\sim 6 \mathrm{e}^{-}$, slightly lower than in the previous case due to the lower temperature, and increases to $\sim 11 \mathrm{e}^{-}$for the sample irradiated at $3 \cdot 10^{15} 1 \mathrm{MeV} \mathrm{n} \mathrm{n}_{\mathrm{eq}} / \mathrm{cm}^{2}$ and 3 Mrad. The lack of substantial non-Gaussian tails suggests a negligible contribution of RTS noise. The pixel-to-pixel variation increases from $\sim 7 \mathrm{e}^{-}$in the unirradiated case to $\sim 12.5 \mathrm{e}^{-}$for the sample irradiated at $3 \cdot 10^{15} 1 \mathrm{MeV} \mathrm{n} \mathrm{n}_{\mathrm{eq}} / \mathrm{cm}^{2}$ and 3 Mrad.

To evaluate the front-end performance for higher TID levels, the chip has been irradiated with X-rays at a dose rate of $25 \mathrm{krad} / \mathrm{min}$ up to $100 \mathrm{Mrad}$. The chip still shows full functionality at this TID level. The irradiation was stopped at different doses to perform basic functionality tests and evaluate the front-end performance. In order to reproduce 

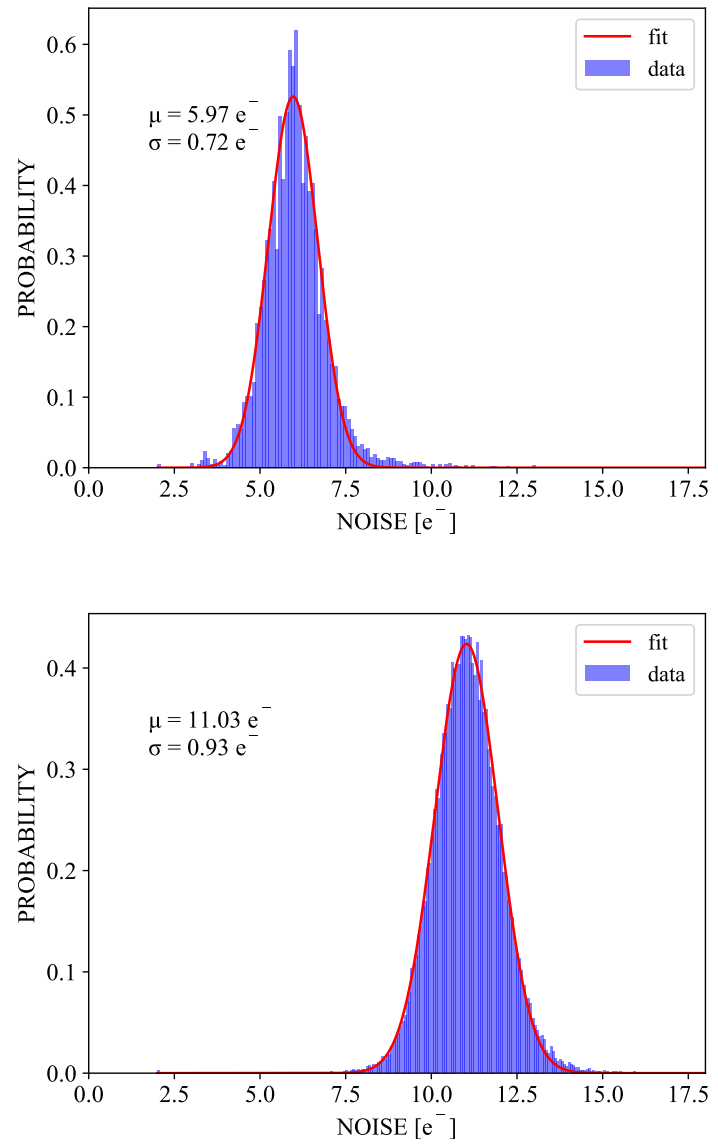

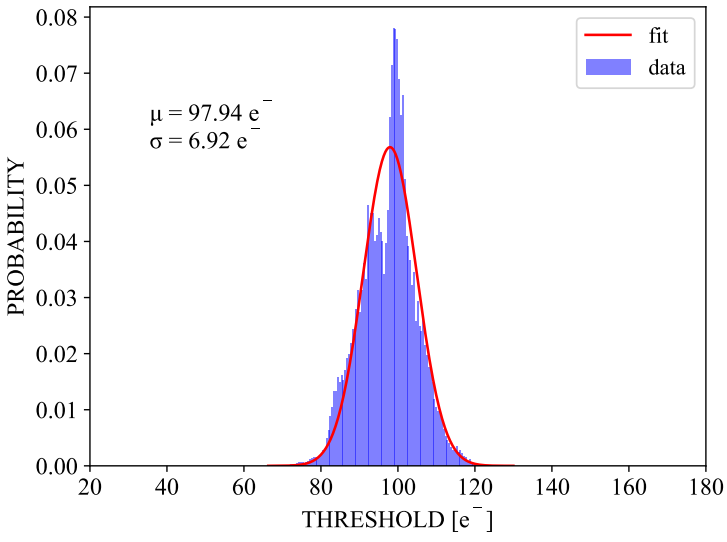

(a)

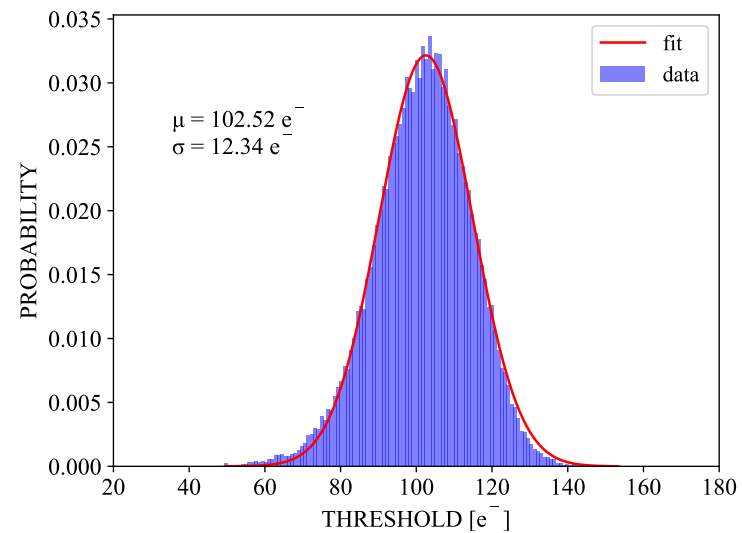

(b)

Fig. 14: Distribution of ENC and threshold with a threshold of $\sim 100 \mathrm{e}^{-}$for (a) an unirradiated sample and (b) a sample irradiated at $3 \cdot 10^{15} 1 \mathrm{MeV} \mathrm{n} \mathrm{n}_{\mathrm{eq}} / \mathrm{cm}^{2}$ and $3 \mathrm{Mrad}$.

the typical operating conditions, the chip was powered and biased during irradiation. Additionally, to minimise annealing effects, the chip was kept at low temperature $\left(-10^{\circ} \mathrm{C}\right)$ during the whole process. The measured ENC and pixel-to-pixel threshold variation as a function of TID are shown in Fig. 15. The threshold was adjusted to $\sim 100 \mathrm{e}^{-}$at each step of the measurement. The ENC grows monotonically from $\sim 5.9 \mathrm{e}^{-}$ before irradiation (first data point) to $\sim 22.5 \mathrm{e}^{-}$at $100 \mathrm{Mrad}$. High levels of RTS noise are present in the ENC distributions for TID levels higher than 1 Mrad. However, already after 24 hours of annealing at room temperature, the RTS noise disappears and the mean ENC drops from $\sim 22.5 \mathrm{e}^{-}$to $\sim 19 \mathrm{e}^{-}$(data point at $250 \mathrm{Mrad}$ ). After other 24 hours of annealing at $80{ }^{\circ} \mathrm{C}$, the mean ENC reduces to $\sim 14 \mathrm{e}^{-}$(last data point). As for the pixel-to-pixel threshold variation, it increases from $\sim 6.8 \mathrm{e}^{-}$before irradiation to $\sim 23 \mathrm{e}^{-}$at $100 \mathrm{Mrad}$. The threshold dispersion more rapidly increases with TID compared to the noise, but it settles around $\sim 23$ $\mathrm{e}^{-}$already at 1 Mrad. After 24 hours of annealing at room temperature, it drops to $\sim 14.5 \mathrm{e}^{-}$and it further drops to $\sim 9$ $\mathrm{e}^{-}$after other 24 hours of annealing at $80{ }^{\circ} \mathrm{C}$.

\section{CONClusion}

This paper describes the design and characterization of a low-power, radiation-hard front-end circuit for monolithic pixel sensors. The circuit is implemented in the TowerJazz 180 nm CMOS imaging technology and integrated in the MALTA2 chip. The sensor features a small octagonal collection electrode with a diameter of $2 \mu \mathrm{m}$ to achieve a low sensor capacitance $(<5 \mathrm{fF})$, key for low-power operation. Process modifications have been introduced to fully deplete the sensor and enhance the lateral electric field in the pixel corners for good tolerance to NIEL. The front-end is a continuously active open-loop amplifier followed by a high-gain common-source discriminator stage. It is designed for a gain of $\sim 2 \mathrm{mV} / \mathrm{e}^{-}$and a peaking time in the order of tens of ns, requiring $<1 \mu \mathrm{W}$ per pixel and $\sim 160 \mu \mathrm{m}^{2}$. It is optimized for low noise and low pixel-to-pixel variation to achieve low charge thresholds. MALTA2 samples were extensively characterized with charge injection tests and radiation source measurements to evaluate the front-end performance before and after irradiation. The signal obtained at the amplifier output during exposure to a ${ }^{55} \mathrm{Fe}$ isotope is higher for neutron-irradiated samples due to a combination of sustained high gain and reduced sensor capacitance. The main front-end metrics with a threshold of 100 $\mathrm{e}^{-}$are summarized in Table II. Compared to a conventional charge-sensitive amplifier using a feedback capacitor, for a comparable power consumption and sensor capacitance [23], this front-end has a 2 times lower ENC and a 5 times lower threshold dispersion. The small collection electrode offers a 


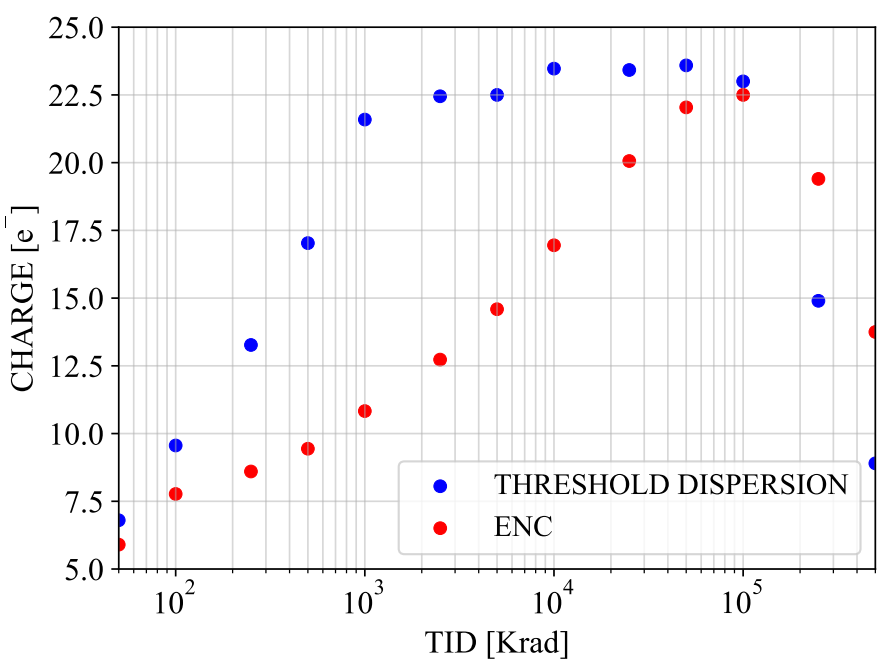

Fig. 15: Dependence of ENC and threshold dispersion on TID with a threshold of $\sim 100 \mathrm{e}^{-}$. The points at $50 \mathrm{krad}$ represent measurements before irradiation. The points at 250 Mrad and 500 Mrad correspond to measurements after 24 hours annealing at room temperature and additional 24 hours of annealing at $80{ }^{\circ} \mathrm{C}$, respectively.

small sensor capacitance and reduces the front-end power consumption by an order of magnitude for the same target time resolution compared to large-electrode designs [24], and therefore constitutes a more power-efficient approach.

TABLE II: Front-end specifications with a $100 \mathrm{e}^{-}$threshold

\begin{tabular}{|c|c|c|}
\hline \multicolumn{2}{|r|}{ Parameter } & Value \\
\hline \multicolumn{2}{|r|}{ Area } & $160 \mu \mathrm{m}^{2}$ \\
\hline \multicolumn{2}{|r|}{ Power consumption } & $1 \mu \mathrm{W}$ \\
\hline \multicolumn{2}{|c|}{ In-time threshold (for a $25 \mathrm{~ns}$ time window) } & $200 \mathrm{e}^{-}$ \\
\hline \multirow{3}{*}{ ENC } & unirradiated & $6.5 \mathrm{e}^{-}$ \\
\hline & $3 \cdot 10^{15} 1 \mathrm{MeV} \mathrm{n} \mathrm{eq} / \mathrm{cm}^{2}, 3 \mathrm{Mrad}$ & $11 \mathrm{e}^{-}$ \\
\hline & $100 \mathrm{Mrad}$ & $22.5 \mathrm{e}^{-}$ \\
\hline \multirow{3}{*}{$\begin{array}{l}\text { Threshold } \\
\text { dispersion }\end{array}$} & unirradiated & $6 \mathrm{e}^{-}$ \\
\hline & $3 \cdot 10^{15} 1 \mathrm{MeV} \mathrm{n} \mathrm{eq} / \mathrm{cm}^{2}, 3 \mathrm{Mrad}$ & $12.5 \mathrm{e}^{-}$ \\
\hline & $100 \mathrm{Mrad}$ & $23 \mathrm{e}^{-}$ \\
\hline \multirow{2}{*}{ Timing jitter } & at threshold & $4.7 \mathrm{~ns}$ \\
\hline & for high charges $\left(\gtrsim 1200 \mathrm{e}^{-}\right)$ & $0.16 \mathrm{~ns}$ \\
\hline
\end{tabular}

\section{ACKNOWLEDGMENT}

The authors are grateful to the Jožef Stefan Institute, Ljubljana, Slovenia for the support during the neutron irradiation.

This project has received funding from the European Unions Horizon 2020 research and innovation programme under grant agreement No. 675587.

\section{REFERENCES}

[1] H. Pernegger, et al., "First tests of a novel radiation hard CMOS sensor process for Depleted Monolithic Active Pixel Sensors," J. Instrum., vol. 12, no. 6, Art. no. P06008, 2017, doi: 10.1088/1748-0221/12/06/p06008.
[2] ATLAS Collaboration, "Technical Design Report for the ATLAS Inner Tracker Pixel Detector," CERN-LHCC-2017-021, ATLAS-TDR-030, Sep. 2017, doi: https://cds.cern.ch/record/2285585.

[3] I. Berdalovic, et al., 'Monolithic pixel development in TowerJazz 180 $\mathrm{nm}$ CMOS for the outer pixel layers in the ATLAS experiment," $J$. Instrum., vol. 13, no. 1, Art. no. C01023, 2018, doi: 10.1088/17480221/13/01/c01023.

[4] I. Berdalovic, et al., 'MALTA: a CMOS pixel sensor with asynchronous readout for the ATLAS High-Luminosity upgrade," in IEEE Nuclear Science Symp. and Medical Imaging Conf., Nov. 2018, doi: 10.1109/NSSMIC.2018.8824349.

[5] E. Schioppa, et al., "Measurement results of the MALTA monolithic pixel detector," Nucl. Instrum. Methods Phys. Res. A, vol. 958, p. 162404, 2020, doi: https://doi.org/10.1016/j.nima.2019.162404.

[6] M. Dyndal, et al., "Mini-MALTA: radiation hard pixel designs for smallelectrode monolithic CMOS sensors for the High Luminosity LHC,' J. Instrum., vol. 15, no. 2, Art. no. P02005, 2020, doi: 10.1088/17480221/15/02/p02005.

[7] S. Meroli, et al., "Energy loss measurement for charged particles in very thin silicon layers," J. Instrum., vol. 6, no. 6, Art. no. P06013, 2011, doi: 10.1088/1748-0221/6/06/p06013.

[8] W. Snoeys, et al., "A process modification for CMOS monolithic active pixel sensors for enhanced depletion timing performance and radiation tolerance," Nucl. Instrum. Methods Phys. Res. A, vol. 871, pp. 90-96, 2017, doi: https://doi.org/10.1016/j.nima.2017.07.046.

[9] M. Munker, et al., "Simulations of CMOS pixel sensors with a small collection electrode, improved for a faster charge collection and increased radiation tolerance," J. Instrum., vol. 14, no. 5, Art. no. C05013, 2019, doi: 10.1088/1748-0221/14/05/c05013.

[10] W. Snoeys, "Monolithic pixel detectors for high energy physics," Nucl. Instrum. Methods Phys. Res. A, vol. 731, pp. 125-130, 2013, doi: https://doi.org/10.1016/j.nima.2013.05.073.

[11] M. Munker, "Test beam and simulation studies on High Resistivity CMOS pixel sensors," Ph.D. dissertation, University of Bonn, Germany, 2018, doi: urn:nbn:de:hbz:5n-51913.

[12] H. Hillemanns, et al., "Radiation hardness and detector performance of new 180nm CMOS MAPS prototype test structures developed for the upgrade of the ALICE Inner Tracking System," in IEEE Nuclear Science Symp. and Medical Imaging Conf., Oct. 2013, doi: 10.1109/NSSMIC.2013.6829475.

[13] F. Krummenacher, "Pixel detectors with local intelligence: an IC designer point of view," Nucl. Instrum. Methods Phys. Res. A, vol. 305, pp. 527-532, 1991, doi: https://doi.org/10.1016/0168-9002(91)90152-G.

[14] C. Gao, et al., "A novel source-drain follower for monolithic active pixel sensors," Nucl. Instrum. Methods Phys. Res. A, vol. 831, pp. 147-155, 2016, doi: https://doi.org/10.1016/j.nima.2016.03.074.

[15] M. J. M. Pelgrom, et al., "Matching properties of MOS transistors," IEEE J. Solid-State Circuits, vol. 24, pp. 1433-1439, 1989, doi: 10.1109/JSSC.1989.572629.

[16] F. Faccio, et al., "Radiation-Induced Short Channel (RISCE) and Narrow Channel (RINCE) Effects in 65 and $130 \mathrm{~nm}$ MOSFETs," IEEE Trans. on Nuclear Science, vol. 62, pp. 2933-2940, 2015, doi: 10.1109/TNS.2015.2492778.

[17] H. Hillemanns, et al., "Total ionizing dose effects in $180 \mathrm{~nm} \mathrm{CMOS}$ structures for monolithic active pixel sensors for the ALICE Inner Tracking System detector upgrade," ALICE internal note, Nov. 2013.

[18] W. Snoeys, et al., "A new NMOS layout structure for radiation tolerance," in IEEE Nuclear Science Symp. Conf. Rec., vol. 2, pp. 822-826, 2001, doi: 10.1109/NSSMIC.2001.1009683.

[19] R. Cardella, et al., "LAPA, a $5 \mathrm{~Gb} / \mathrm{s}$ modular pseudo-LVDS driver in $180 \mathrm{~nm}$ CMOS with capacitively coupled pre-emphasis," Proc. Sci., vol. 313, p. 038, 2017, doi: https://doi.org/10.22323/1.313.0038.

[20] L. Perktold, et al., "A multichannel time-to-digital converter ASIC with better than 3 ps RMS time resolution," J. Instrum., vol. 9, no. 1, Art. no. C01060, 2014, doi: 10.1088/1748-0221/9/01/c01060.

[21] L. Snoj, et al., "Computational analysis of irradiation facilities at the JSI TRIGA reactor," Applied Radiation and Isotopes, vol. 70, pp. 483-488, 2012, doi: https://doi.org/10.1016/j.apradiso.2011.11.042.

[22] L. Rossi, et al., "Pixel Detectors: From Fundamentals to Applications," Berlin, Springer, 2006, ISBN: 978-3-642-06652-8, p. 35.

[23] I. Kremastiotis, et al., "Design and Characterization of the CLICTD Pixelated Monolithic Sensor Chip," IEEE Trans. Nucl. Sci., vol. 67, no. 10, pp. 2263-2272, 2020, doi: 10.1109/TNS.2020.3019887.

[24] R. Schimassek, et al., "Test results of ATLASPIX3 - A reticle size HVCMOS pixel sensor designed for construction of multi chip modules," Nucl. Instrum. Methods Phys. Res. A, vol. 986, p. 164812, 2021, doi: https://doi.org/10.1016/j.nima.2020.164812. 